\title{
Enhanced resolution induced by random silver nanoparticles in near-field optical disks
}

\author{
Tai Chi Chu ${ }^{\text {a,b }}$, Wei-Chih Liu ${ }^{\text {c,* }}$, Din Ping Tsai ${ }^{\text {a,b }}$ \\ ${ }^{a}$ Center for Nanostorage Research, National Taiwan University, Taipei 10617, Taiwan \\ ${ }^{\mathrm{b}}$ Department of Physics, National Taiwan University, Taipei 10617, Taiwan \\ ${ }^{c}$ Department of Physics, National Taiwan Normal University, Taipei 11676, Taiwan
}

Received 27 July 2004; received in revised form 29 October 2004; accepted 1 November 2004

\begin{abstract}
The super-resolution near-field structure (super-RENS) is a high-density near-field optical data storage medium which can achieve superior spatial resolution beyond the diffraction limit. Our previous studies found that enhanced local optical intensity occurred at the near fields of super-RENS disks, and the nonlinear near-field optical enhancement is related to the localized surface plasmons of silver clusters dissociated from the $\mathrm{AgO}_{x}$ layer in the super-RENS disks. In this paper, we studied the near-field and far-field properties of $\mathrm{AgO}_{x}$-type super-RENS with different distributions of silver nanoparticles using finite-difference time-domain (FDTD) simulations. Highly localized enhancements are found between adjacent silver nanoparticles in the near fields. The far-field signals of different silver nanoparticles distributions confirm the super-resolution capability of $\mathrm{AgO}_{x}$-type Super-RENS disks, and a simplified Fourier optics model is used to describe the relation between highly localized near-field distributions and enhanced resolution of far-field signals.
\end{abstract}

(c) 2004 Elsevier B.V. All rights reserved.

\section{Introduction}

Near-field optical data storage technique was first demonstrated by Betzig et al. [1,2] on multi-

\footnotetext{
${ }^{*}$ Corresponding author. Tel.: +88622934 3183; fax: +8862 29326408.

E-mail address: wcliu@phy.ntnu.edu.tw (W.-C. Liu).
}

layered Pt/Co magneto-optical thin films using a near-field scanning optical microscope (NSOM). High spatial resolution recording was accomplished by using a sub-wavelength aperture at the tapered tip of an optical fiber operated in the near-field region. Though the diffraction limit is successfully overcome, it is difficult to precisely 
control the distance between probe and medium less than a wavelength and to simultaneously maintain a high scanning rate. The recording speed of near-field optical recording using NSOM is still around $\mu \mathrm{m} / \mathrm{s}$, which is quite slow comparing with the recording speed of a commercial digital versatile disk (DVD), which is higher than $3.5 \mathrm{~m} / \mathrm{s}$. Terris et al. [3] used solid immersion lens (SIL) to reduce the mechanical damage which comes from the near-field optical fiber probe, and to achieve higher recording speed. Although the focused spot size is decreased to $317 \mathrm{~nm}$, the control of near-field distance between the SIL recording head and the recording medium remains major difficulties for commercial applications.

In 1998, the multilayer structure, polycarbonate/ $\mathrm{SiN}(170 \mathrm{~nm}) / \mathrm{Sb}(15 \mathrm{~nm}) / \mathrm{SiN}(20 \mathrm{~nm}) / \mathrm{GeSbTe}(15 \mathrm{~nm}) /$ $\mathrm{SiN}(20 \mathrm{~nm})$, was demonstrated to overcome the diffraction limits and the $\mathrm{SiN} / \mathrm{Sb} / \mathrm{SiN}$ layer was named super-resolution near-field structure (superRENS) [4-7]. The carrier-to-noise ratio (CNR) is more than $10 \mathrm{~dB}$ with $90 \mathrm{~nm}$ recording marks. The thin Sb film above the phase-change medium, GeS$\mathrm{bTe}$, in the super-RENS replaces the fiber probe in the near-field optical recording system. Subsequently, the $\mathrm{AgO}_{x}$-type super-RENS [8] was developed for higher carrier-to-noise ratio (CNR). For $90 \mathrm{~nm}$ recording marks, the CNR reached to $15 \mathrm{~dB}$. To understand the optical properties of the $\mathrm{AgO}_{x}$-type super-RENS, we have used a tappingmode tuning-fork NSOM in transmission mode to measure the near-field optical images [9-11], and found that highly localized near-field intensity enhancements are occurred around the superRENS. Our previous studies have also shown that enhanced local optical intensity exhibits at the near field, and the unusual optical effect is related to the localized surface plasmon of silver clusters dissociated from $\mathrm{AgO}_{x}$ [11-16]. To study the relation between random silver nanoparticles and nonlinear localized enhancements, the near-field optical fields of $\mathrm{AgO}_{x}$-type super-RENS with silver nanoparticles embedded in $\mathrm{AgO}_{x}$ layer were calculated by two-dimensional finite-difference time-domain (FDTD) simulations $[11,15,16]$. The silver nanoparticles and the localized evanescent waves around silver nanoparticles could act as virtual near-field optical probes and the disadvantages of the con- ventional SNOM were solved by the super-RENS technology.

Metallic nanoparticles of subwavelength size exhibit plenty of optical phenomena related to geometry-dependent surface plasmon resonances. The enhanced electric fields are confined within a few nanometers near the surface of nanoparticles. The resonances are due to dipolar or high-polar collective oscillations of conduction electrons in particles $[17,18]$. In previous studies, we found that the super-resolution efficiency of the $\mathrm{AgO}_{x}$-type super-RENS is related to the localized surface plasmon of silver clusters dissociated from the $\mathrm{AgO}_{x}$ layer and the complicated interactions between the nonlinear near-field optical enhancements and the subwavelength recording marks [11-16]. The highly enhanced localized surface plasmons improve the reading efficiency, but the relation between the nonlinear near-field optical effects and the detective far-field signals is still not clear. To further understand the unusual optical effect of the $\mathrm{AgO}_{x}$-type super-RENS, we used 2D FDTD numerical simulation method to study the near-field properties of the $\mathrm{AgO}_{x}$-type superRENS disks with different random distributions of silver nanoparticles. Since the detectable optical signals are the far-field intensities, the near-to-far field transformation [19] was used to realize the relations between near-field enhancements and far-field variations of the $\mathrm{AgO}_{x}$-type super-RENS disks with different distributions of embedded silver nanoparticles.

\section{Simulation model}

The numerical method is a two-dimensional FDTD with periodic boundary condition and perfect matched layers, which have the advantages of reduced memory requirement in computation, and simple process in handling the complex structure. The structure of the super-RENS disk was cover glass $/ \mathrm{ZnS}-\mathrm{SiO}_{2}(20 \mathrm{~nm}) / \mathrm{AgO}{ }_{x}(15 \mathrm{~nm}) / \mathrm{ZnS}-\mathrm{SiO}_{2}$ $(20 \mathrm{~nm}) / \mathrm{GeSbTe}(16 \mathrm{~nm})$, as shown in Fig. 1 . The incident laser light was Gaussian distributed with wavelength of $650 \mathrm{~nm}$, and the numerical aperture of the lens was 0.85 . The refractive index of $\mathrm{ZnS}-\mathrm{SiO}_{2}$ and $\mathrm{AgO}_{x}$ layer was 2.07 and 2.7, 
respectively. Recording marks were created by the laser beam focused on the phase changed recording medium $\mathrm{Ge}_{2} \mathrm{Sb}_{2} \mathrm{Te}_{5}$ (GST), and the refractive indices of amorphous GST and crystalline recording mark were $3.38+4.07 \mathrm{i}$ and $3.855+4.80 \mathrm{i}$, respectively [16]. The marks spread periodically in a $4000 \mathrm{~nm}$ regime, while the period of marks was double of the mark length. Silver nanoparticles and clusters embedded in the $\mathrm{AgO}_{x}$ layer were decomposed from $\mathrm{AgO}_{x}$ during the recording process, and the refractive index of the silver nanoparticles was $0.055+4.44$ i.

The distribution of the dissociated silver nanoparticles and clusters is very difficult to be observed directly. From experimental results, we found two possible distributions of the nanoparticles or clusters at the $\mathrm{AgO}_{x}$ layer of the super-

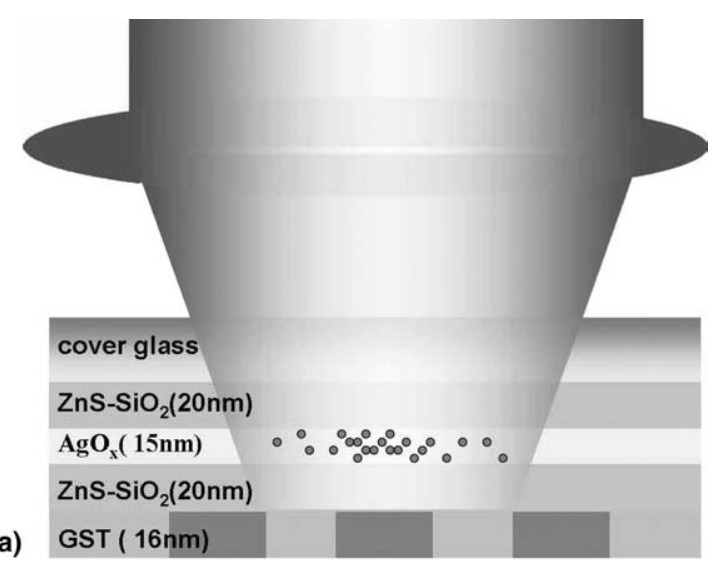

(a)

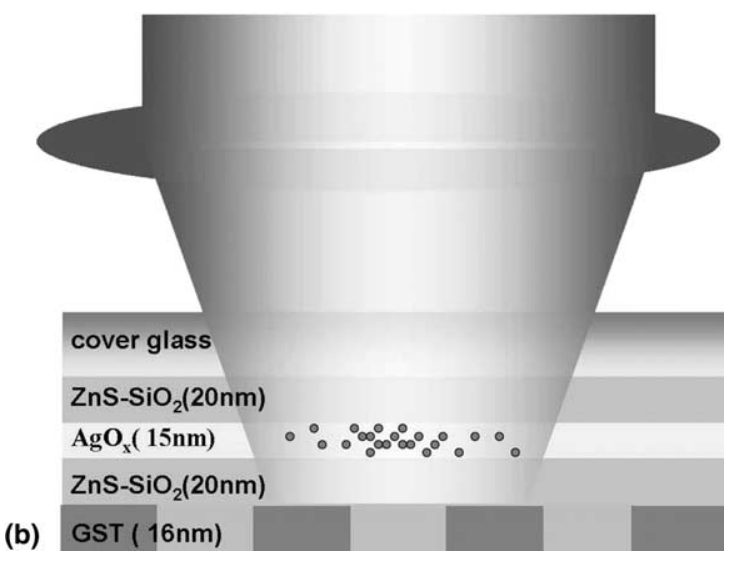

Fig. 1. (a) Structure of the super-RENS disk with embedded silver nanoparticles for on-mark situations; (b) off-mark situations.
RENS [20]. The reading or recording focused laser beam drove the dissociated silver nanoparticles to form aggregative silver clusters or diffused ring structure. Experimental results have also showed that the intensity and distribution of dissociated silver nanoparticles was influenced by the intensity of focused laser spot. The density of silver nanoparticles or clusters was assumed to increase with density of incident light, and optical properties of these two cases were compared in this paper. For the aggregative case, distribution of nanoparticles was set to be a random Gaussian distribution. The distribution function of the random Gaussian distribution is $\exp \left(-\left(Y-Y_{0}\right)^{2} /\left(2 \sigma^{2}\right)\right)$, where $Y_{0}$ is the center position of the focus beam, and $\sigma$ is $416 \mathrm{~nm}$ which is the full wave half width of the focus spot. The amount of silver nanoparticles was 100 and 200, and they were spread in a $4000 \mathrm{~nm}$ $(\times 15 \mathrm{~nm})$ regime. For another case, the silver particles were driven by incident laser pulse to form a ring structure, and we assumed that silver particles formed two Gaussian distributions at the edges of the incident laser beam. The number of nanoparticles was 100 and 200, and they were spread in a total $4000 \mathrm{~nm}$ regime. In this paper, we concentrated on the effects of the random distribution of nanoparticles, so size variation of silver particles was neglected. The diameter of silver nanoparticles was $4 \mathrm{~nm}$ and the dispersive behavior of silver and GST was simulated by the Lorentz model [21] and the material parameters used in our simulation were listed in Table 1.

To study the far-field optical propertied induced by the enhancements of localized surface plasmons, a near-to-far transformation was used to obtain the far-field response from near-field results $[19,21]$. The method is accurate within the limits of the Kirchoff approximation. A Fourier transform is applied to the fields in the sampling plane to obtain the angular distribution of the radiation in the far-field. An efficient transform expression to get the angular distribution of the electric fields in the far-field is given by the integral expression

$$
\begin{aligned}
E_{y}^{\mathrm{scat}}(R, \theta)= & \frac{1}{2 \sqrt{\lambda R}} \int_{\text {sampling plane }} \mathrm{d} y^{\prime} \exp \left[\mathrm{j} k_{0} \sin (\theta) y^{\prime}\right] \\
& \times\left[E_{y}\left(x, y^{\prime}\right)+\eta H_{z}\left(x, y^{\prime}\right) \cos \theta\right]
\end{aligned}
$$


Table 1

Material parameters used in the simulation for the Lorentz model [21]

\begin{tabular}{lllll}
\hline Material & $\mathrm{AgO}_{x}$ & $\mathrm{Ag}$ & GST (normal) & GST (mark) \\
\hline Resonant frequency, $\omega_{0}\left(\mathrm{~s}^{-1}\right)$ & $5.097 \times 10^{15}$ & $2.41477 \times 10^{15}$ & $3.57 \times 10^{15}$ & $2.9739 \times 10^{15}$ \\
Damping coefficient, $\Gamma\left(\mathrm{s}^{-1}\right)$ & $59.57 \times 10^{13}$ & $1.9378 \times 10^{13}$ & $1.548 \times 10^{15}$ & $1.1844 \times 10^{15}$ \\
\hline
\end{tabular}

The DC response of the polarization to the electric field $\chi_{0}=10$ and the instantaneous permittivity $\varepsilon_{\infty}=2.89 \varepsilon_{0}$.

where $\theta$ is the discrete angle defined by the normal to the incident plane and the observation point at the detector on a circle of radius $R$. The intensities are integrated within the numerical aperture of the objective lens.

In order to correlate the simulation results with the experiment results, the far-field intensity differences between the on-mark and the off-mark cases (shown in the Fig. 1) were calculated for various mark sizes. The on-mark situation meant that the incident light focuses on the recording mark, and off-mark situation meant that the incident light focuses between two marks. The far-field intensity differences between on-mark and off-mark situations was calculated by $10 * \log \left(\left|I_{\text {on }}-I_{\text {off }}\right| / I_{\text {no-mark }}\right)$, where $I_{\text {on }}$ is the far-field intensity for the on-mark case, $I_{\mathrm{off}}$ is the far-field intensity for the off-mark case, $I_{\text {no-mark }}$ is the far-field intensity for the case without any marks. The far-field difference signals could demonstrate the capability of super-RENS disks to distinguish subwavelength recording marks.

\section{Results and discussion}

Near-field distributions from two-dimensional FDTD simulation are shown in Fig. 2. Highly localized enhancements were produced between adjacent silver nanoparticles in the near field. The localized enhancements induced by the silver nanoparticles of random Gaussian distribution were similar with that of ring distribution. These near-field optical signals of the $\mathrm{AgO}_{x}$ thin film corresponded to the nonlinear near-field optical properties which were previously found in experiments [10].

There were no significant differences between the near-field optical responses of random Gaussian and ring distribution, and the localized enhancements were strongly influenced by the densities of silver nanoparticles [16]. In order to compare the simulation results to the measured carrier-to-noise ratio (CNR) in an optical disk tester, the far-field intensity differences between the on-mark and the off-mark situations of the super-RENS disks were computed for the various mark sizes. Fig. 3(a) shows the far-field intensity differences for the dissociated silver nanoparticles of random Gaussian distribution. These results were normalized against the far-field signals of the $\mathrm{Ge}_{2} \mathrm{Sb}_{2} \mathrm{Te}_{5}$ recording layer without recording marks. In the case of no silver nanoparticles, the far-field signals dropped around $200 \mathrm{~nm}$ of the mark size and it subjected to the diffraction limit. All the cases with random silver nanoparticles embedded in the $\mathrm{AgO}_{x}$ layer confirmed the super-resolution capability and the far-field signals of mark size smaller than $100 \mathrm{~nm}$ were distinguishable. Similar results have been observed in our previous CNR measurements of the $\mathrm{AgO}_{x}$-type super-RENS disks $[10,11]$. The signal enhancement in the super-RENS disks beyond diffraction limit was clearly demonstrated in our simulation results. For the silver nanoparticles of ring distribution, the far-field signals are shown in Fig. 3(b). Similar super-resolution properties were obvious for both the cases of 100 and 200 nanoparticles. The distribution of embedded silver nanoparticles influenced the near-field enhancements, so the trend of far-field signals of different cases had slight variance. However, the far-field signals of the recording marks beyond the diffraction limit indicated a kind of the saturation behavior when the number of the silver nanoparticles increased above a threshold. A distinct nonlinear response of the $\mathrm{AgOx}$ thin film was shown in the results of our simulation, which agreed with our previous near-field and farfield measurements.

The plasmon resonance of small individual metallic particles is a well-known phenomenon and this resonance plays an important role in sur- 

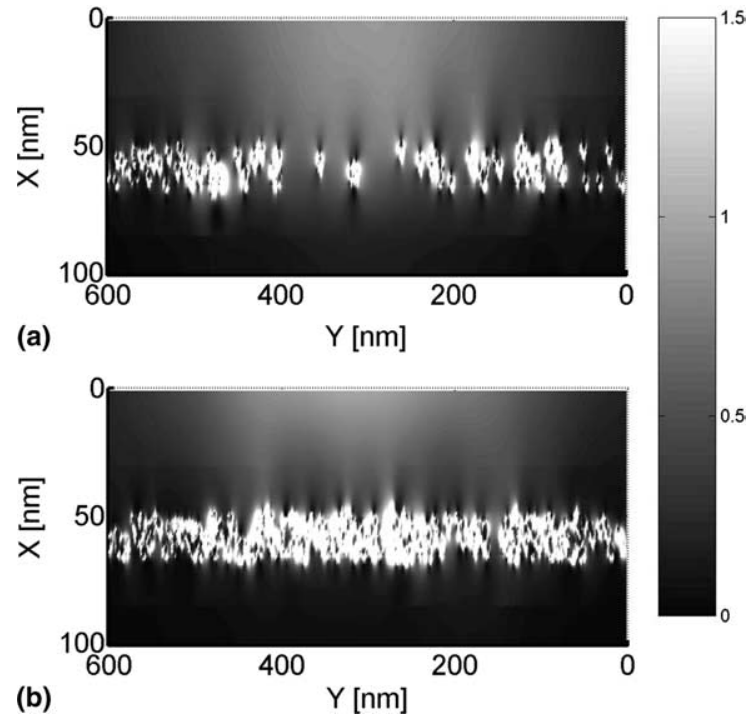

Fig. 2. Near-field intensity distributions from FDTD simulation for silver nanoparticles of: (a) Gaussian distribution; (b) ring distribution.

face enhanced Raman scattering (SERS). When two or more nanoparticles are brought together, additional resonances for coupled system are excited. These plasmon resonances are the interaction between incident electromagnetic waves and induced charges in metallic particles. With a p-polarized illuminated wave (also called TM polarization; no plasmon resonance can be excited for the s-polarization case), polarization charges are induced in the coupled system by the external field and interact with the illuminated waves. The coupling resonances between nanoparticles are influenced by induced charge distribution of clusters, unless the nanoparticles are well separated with one another. In the case of our simulation model, the random distribution of silver nanoparticles was Gaussian and the density of silver nanoparticles was higher at the center of the focused beam. The incident fields interacted with the overall polarization charges spread on silver clusters and the individual resonance of each random silver nanoparticle was unapparent in comparison with coupling resonances of random silver clusters. So the distribution of polarization charges played a major role in our cases. The distributions of induced charges were similar while the silver nano-
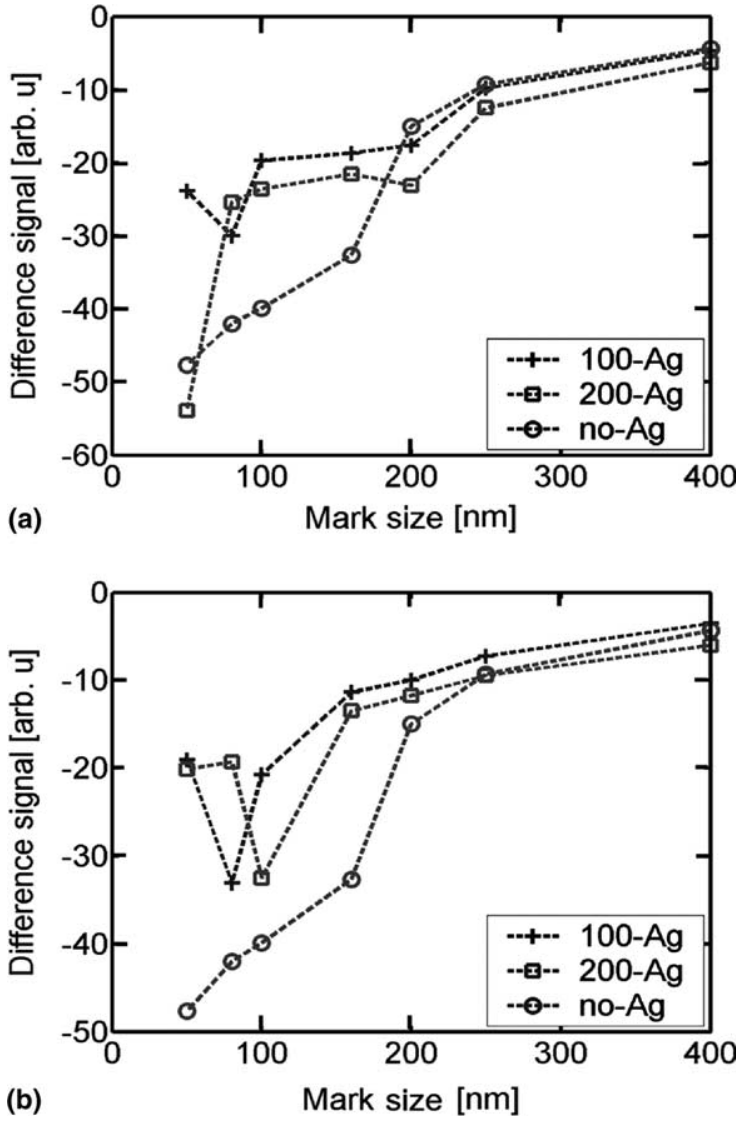

Fig. 3. (a) Far-field signals of Gaussian distribution, and mark sizes are changed from 50 to $400 \mathrm{~nm}$ with 100 silver particles (+), 200 silver particles $(\square)$ and no silver particle (O); (b) farfield signals of ring distribution with 100 silver nanoparticles $(+), 200$ silver particles $(\square)$ and no silver particle $(O)$.

particles act as silver clusters with Gaussian-like distribution and the amount of silver nanoparticles was not a key factor in the coupled resonances.

The enhanced evanescent waves of silver nanoparticles were localized and these localized effects influence the detected far-field signals. A simplified explanation based on Fourier optics of subwavelength structures [22-24] was used to realize the simulation results. The far-field spatial spectrum was the convolution of the reflected light from recording marks and the optical function of $\mathrm{AgO}_{x}$ layer in super-RENS $[25,26]$. Although the spatial spectrum of the embedded silver particles was random, the enhanced evanescent components coupled into the propagating component in the 


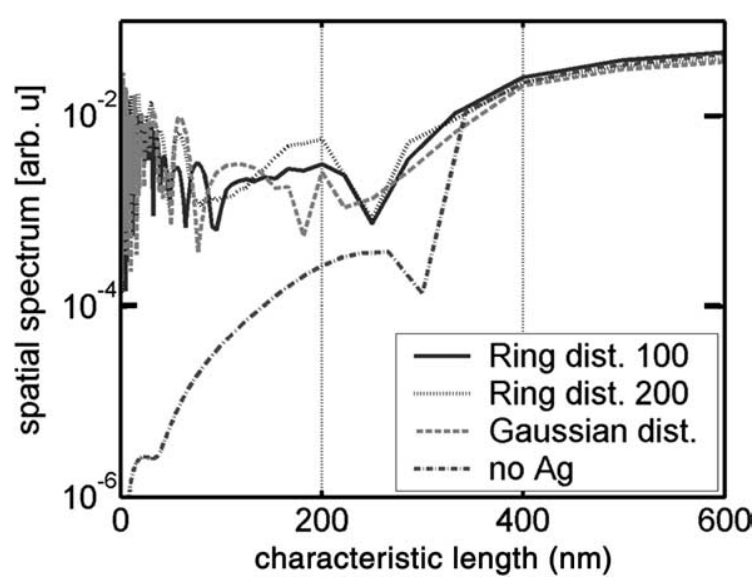

Fig. 4. Reflection spatial spectra at the boundary of the $\mathrm{AgO}_{x}$ layer with Gaussian distribution and Ring distribution silver nanoparticles.

reflected light. Fig. 4 shows the reflection spatial spectra of the near-field simulation results which were sampled at the boundary of $\mathrm{AgO}_{x}$ layer and were plotted as functions of characteristic length $l=2 \pi / k$. The reflected spatial spectra are the Fourier transform of the electric fields at the boundary at the boundary of $\mathrm{AgO}_{x}$ layer for the cases without any marks. To simplify the situation, we used the cases of no recording marks in the GeSbTe layer to compare the spatial spectra. In the case of no silver nanoparticles embedded, the spatial spectrum dropped rapidly around the characteristic length of $300 \mathrm{~nm}$ which indicate the reduction of far-field signals under diffraction limit. For the cases of various distributions of silver nanoparticles, the spatial spectra were broad and randomly enhanced with characteristic length smaller than $300 \mathrm{~nm}$. The behavior was consistent with the farfield results shown in Fig. 2. However, the far-field signals were the convolution between the reflected light from recording marks and the optical function of the $\mathrm{AgO}_{x}$ layer. Although the spatial spectrum indicated the super-resolution capability shown in Fig. 3, the trend of spatial spectra was not exactly the same as that of far-field signals.

From above Fourier optics explanation, the enhanced evanescent waves from the embedded silver nanoparticles enhanced the far-field signals. We compared the transmittance intensity spectra of 8 and $20 \mathrm{~nm}$ away from the $\mathrm{AgO}_{x}$ layer to illustrate the character of the evanescent waves of the $\mathrm{AgO}_{x}$ layer. Fig. 5(a) shows the transmittance intensity spectra of $8 \mathrm{~nm}$ away from the $\mathrm{AgO}_{x}$ layer. All the cases with silver nanoparticles embedded showed the strongly enhancements, and the peak intensity of ring distribution with 200 silver nanoparticles was two times stronger than another cases. From the near-field intensity spectra, no obviously correlations were observed between the distribution of silver nanoparticles and intensity spectra. Fig. $5(\mathrm{~b})$ is the transmittance intensity spectra of $20 \mathrm{~nm}$ away from the $\mathrm{AgO}_{x}$ layer, and the strongly enhancements shown in Fig. 5(a) decrease rapidly. It indicated that the local enhancement was indeed from evanescent waves. The enhanced collective evanescent waves almost disappear in $12 \mathrm{~nm}$ from the $\mathrm{AgO}_{x}$ layer, and these
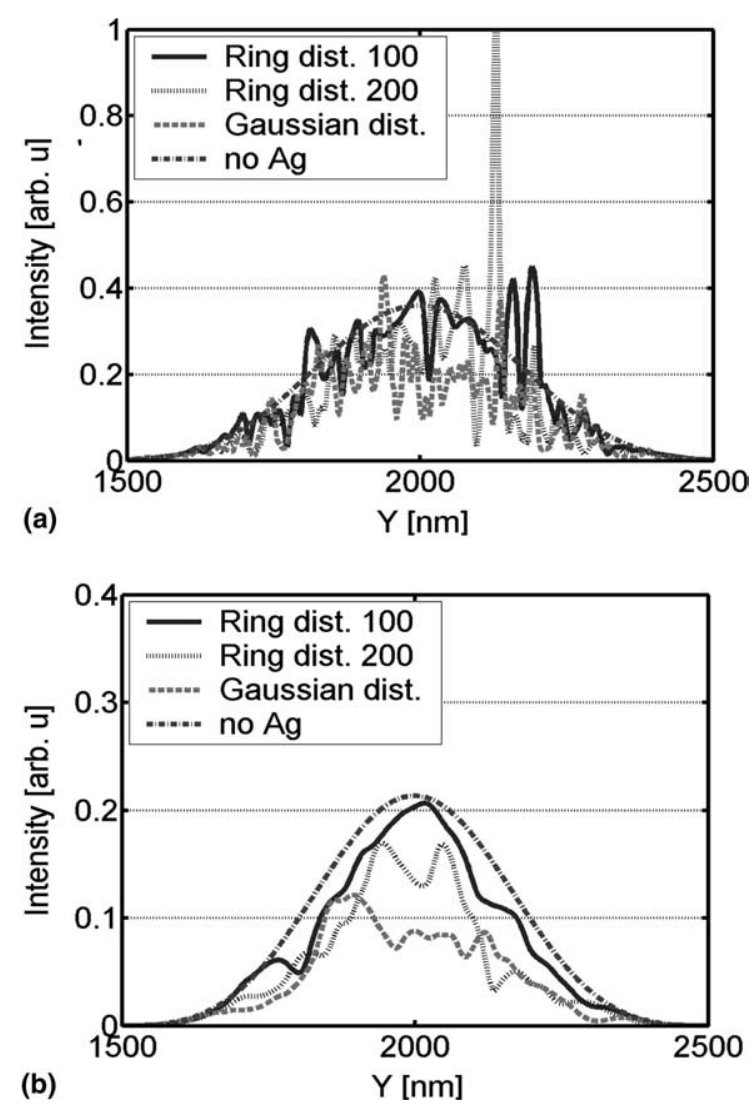

Fig. 5. (a) Transmittance intensity spectra at $8 \mathrm{~nm}$; (b) $20 \mathrm{~nm}$ away from the boundary of the $\mathrm{AgO}_{x}$ layer. 
rapidly decrease phenomenon correspond to the experimental results in the previous studies [10,11].

The near-field and far-field optical properties observed in our simulation results were well consistent with the experimental results of previous studies. Our results demonstrated the localized surface plasmon enhancements induced by the silver nanoparticles result in the far-field super-resolution effects. Although we neglected the size variation in the simulation model, the simulation results yielded a good understanding of the relation between the distribution of embedded silver nanoparticles and the optical properties of the $\mathrm{AgO}_{x}$-type Super-RENS disks.

\section{Conclusions}

In this paper, the near-field and far-field optical properties of the $\mathrm{AgO}_{x}$-type super-RENS results from the complicated interactions between the subwavelength recording marks and randomly distributed silver nanoparticles were studied by FDTD simulations. The far-field intensity difference signals were distinguishable with recording mark size much smaller than the diffraction limit, but for different distributions of random silver nanoparticles, their resolution capabilities had no obvious difference in our simulation results. The near-field results showed the collective enhanced effects were induced by random silver nanoparticles, and there were significant high-frequency components in the spatial spectra of the near fields of the $\mathrm{AgO}_{x}$ layer. The simplified Fourier optical theory provided a theoretical explanation of super-resolution capability of the superRENS disk and identified the evanescent waves enhanced in the near fields of the $\mathrm{AgO}_{x}$ layer induced the super-resolution capability in the far-field signals. Further research on the effects of size variations of the silver nanoparticles may yield a better understanding of the optical properties of $\mathrm{AgO}_{x^{-}}$ type Super-RENS.

\section{Acknowledgement}

This work was supported by the National Science Council and the Ministry of Economical
Affair (93-EC-17-A-08-S1-0006) of Taiwan, Republic of China.

\section{References}

[1] E. Beztig, J.K. Trautman, Science 257 (1992) 189.

[2] E. Betzig, J.K. Trautman, R. Wolfe, E.M. Gyorgy, P.L. Finn, Appl. Phys. Lett. 61 (1992) 142.

[3] B.D. Terris, H.J. Marnin, G.S. Kino, Appl. Phys. Lett. 65 (1994) 388.

[4] J. Tominaga, T. Nakano, N. Atoda, Appl. Phys. Lett. 73 (1998) 2078

[5] J. Tominaga, H. Fuji, A. Sato, T. Nakano, T. Fukaya, N. Atoda, Jpn. J. Appl. Phys. 37 (1998) L1323.

[6] T. Nakano, A. Sato, H. Fuji, J. Tominaga, N. Atoda, Appl. Phys. Lett. 75 (1999) 151

[7] J. Tominaga, H. Fuji, A. Sato, N. Atoda, Jpn. J. Appl. Phys. 39 (2000) 957.

[8] J. Kim, M. Kuwahara, N. Atoda, J. Tominaga, Appl. Phys. Lett. 79 (2001) 2600.

[9] D.P. Tsai, C.W. Yang, W.C. Lin, F.H. Ho, H.J. Huang, M.Y. Chen, T.F. Tseng, C.H. Lee, C.J. Yeh, Jpn. J. Appl. Phys. 39 (2000) 982.

[10] D.P. Tsai, W.C. Lin, Appl. Phys. Lett. 77 (2000) 1413.

[11] W.-C. Liu, C.-Y. Wen, K.-H. Chen, W.C. Lin, D.P. Tsai, Appl. Phys. Lett. 78 (2001) 685.

[12] F.H. Ho, H.H. Chang, Y.H. Lin, D.P. Tsai, Proc. SPIE Int. Soc. Opt. Eng. 4930 (2002) 39.

[13] F.H. Ho, H.H. Chang, D.P. Tsai, P. Wang, Proc. SPIE Int. Soc. Opt. Eng. 4930 (2002) 68.

[14] F.H. Ho, H.H. Chang, Y.H. Lin, B.-M. Chen, S.-Y. Wang, D.P. Tsai, Jpn. J. Appl. Phys. 42 (2003) 1000.

[15] W.-C. Liu, D.P. Tsai, Jpn. J. Appl. Phys. 42 (2003) 1031.

[16] W.-C. Liu, M.-Y. Ng, D.P. Tsai, Jpn. J. Appl. Phys. 43 (2004) 4713.

[17] U. Kreibig, M. Vollmer, Optical properties of metal clusters Springer Series in Materials Science, vol. 25, Springer, Berlin, 1995.

[18] D.P. Tsai, J. Kovacs, Zhouhang Wang, Martin Moskovits, Vladimir M. Shalaev, J.S. Suh, R. Botet, Phys. Rev. Lett. 72 (1994) 4149.

[19] A. Taflove, Computational Electrodynamics, Artech House, Boston-London, 1995, p. 203.

[20] F.H. Ho, H.H. Chang, Y.H. Lin, B.-M. Chen, S.Y. Wang, D.P. Tsai, Jpn. J. Appl. Phys. 42 (2003) 1000 .

[21] J.B. Judkins, R.W. Ziolkowski, J. Opt. Soc. Am. A 12 (1995) 1974.

[22] J.M. Vigoureux, F. Depasse, C. Girard, Appl. Opt. 31 (1992) 3036

[23] J.M. Vigoureux, D. Courjon, Appl. Opt. 31 (1992) 3170.

[24] C.J.R. Sheppard, H. Fatemi, M. Gu, Scanning 17 (1995) 28.

[25] W.-C. Liu, D.P. Tsai, Scanning 26 (2004) I-94.

[26] W.-C. Liu, M.-Y. Ng, D.P. Tsai, Scanning 26 (2004) I-98. 